\title{
Are dieting-related cognitive impairments a function of iron status?
}

\author{
Michael W. Green ${ }^{1,2 *}$ and Nicola A. Elliman ${ }^{1}$ \\ ${ }^{1}$ Aston Nutrition and Behaviour Laboratory, Psychology Department, School of Life and Health Sciences, Aston University, \\ Aston Triangle, Birmingham, B4 7ET, UK \\ ${ }^{2}$ Psychology Department, School of Life and Health Sciences, Aston University, Aston Triangle, Birmingham B4 7ET, UK
}

(Submitted 17 October 2011 - Final revision received 1 February 2012 - Accepted 3 February 2012 - First published online 14 March 2012)

\section{Abstract}

The aim of the present study was to investigate whether the impairments in cognitive function observed in unsupported dieting are related to compromised Fe status. During a non-clinical intervention, overweight participants (age: $18-45$ years, BMI: $25-30 \mathrm{~kg} / \mathrm{m}^{2}$ ) either participated in a commercially available weight-loss regimen ( $n$ 14), dieted without support $(n 17)$ or acted as a non-dieting control group ( $n$ 14) for a period of 8 weeks. Measurements of cognitive function and blood chemistry were taken at a pre-diet baseline, after 1 week and 8 weeks of dieting. After 1 week, unsupported dieters displayed impaired verbal memory, executive function and slower reaction speeds than the other two groups, this difference disappearing by the end of the study. There were no significant group-related changes in blood chemistry over the course of the study, although there were group-related changes in a number of self-reported food-related cognitions. In conclusion, impaired cognition among unsupported dieters is not due to compromised Fe status and is most likely to result from psychological variables.

Key words: Dieting: Dietary support: Cognition: Iron metabolism

Deliberate restriction of food intake to control weight is one of the most common food choice-related behaviour in the developed world. Indeed, over one-third of the general US population is classed as obese ${ }^{(1)}$, with an associated impact on health treatment resources ${ }^{(2)}$. Within the context of these rising obesity rates ${ }^{(3)}$, an understanding of the psychological impact of undernutrition and weight loss assumes an increasing importance.

While there are undoubted health benefits of controlling obesity, the attempt to lose weight by dieting has been associated with a number of negative psychological consequences such as increased depression and low self-esteem ${ }^{(4)}$. Although the evidence concerning the psychological effects of experimentally induced weight loss is equivocal ${ }^{(5)}$, evidence suggests that dieting is reliably associated with a range of impairments in cognitive function. For instance, dieting has been associated with slower reaction speed, impaired verbal memory and an impaired ability to sustain attention ${ }^{(6,7)}$. A number of studies have indicated that this pattern of impaired task performance is primarily due to a reduction in available working memory capacity ${ }^{(8-11)}$, which is primarily due to preoccupying cognitions relating to hunger ${ }^{(8)}$ and body shape-related self-esteem ${ }^{(12,13)}$. This is important, since working memory is the primary cognitive domain, controlling moment-to-moment rules of action, planning ability and the short-term retention of information. The most commonly accepted model of working memory ${ }^{(14)}$ proposes a tripartite system comprising a phonological loop (controlling verbal short-term memory), a visuospatial sketchpad (controlling visual memory) and an overarching supervisory system known as the 'central executive'.

It has also been found that the way in which weight loss is attempted influences cognitive function, with dieting without support being associated with cognitive impairments and increased stress levels (as measured by salivary cortisol secretion) and dieting within the context of an organised weight-loss group not being associated with impaired cognition ${ }^{(15)}$. The present study employed a substantively similar design.

Although the evidence suggests that these negative cognitive consequences of dieting are due to the psychological mechanism noted above (this being supported by the finding of impaired task performance in the absence of any weight loss over the course of the diet $\left.{ }^{(7)}\right)$, there are a number of possible subtle physiological mechanisms which may mediate the effect. It is, therefore, important to investigate the role of these mechanisms in mediating the cognitive impairments associated with spontaneous, unsupervised dieting. It has been shown that total energy restriction per se for periods of up to $24 \mathrm{~h}^{(16)}$ or partial energy restriction in a laboratory-based

Abbreviations: DEBQ, Dutch Eating Behaviour Questionnaire; HADS, Hospital Anxiety and Depression Scale. 
weight-loss programme ${ }^{(17)}$ does not significantly impair task performance. It has also been demonstrated that dietingrelated cognitive impairments cannot be attributed to compromised glucose metabolism ${ }^{(18)}$. It is also a replicable finding that experimentally controlled food deprivation leads to a reduction in plasma levels of the amino acid tryptophan, which is the precursor for the neurotransmitter serotonin (5-hydroxy tryptophan; 5-HT) ${ }^{(19)}$. There is an argument, therefore, to conclude that the impairments in cognition observed in naturalistic dieting are the result of low dietary intakes of tryptophan and, by implication, lower levels of 5-HT synthesis. However, it has been demonstrated that the impairments in cognitive task performance characteristic of unsupported spontaneous dieting are unrelated to 5 -HT function ${ }^{(20)}$.

There is, however, a body of evidence suggesting that $\mathrm{Fe}$ metabolism may be a factor in the impaired cognitive function associated with dieting. Fe deficiency is commonly associated with impaired cognitive function ${ }^{(21)}$. In addition, verylow-energy diets have been associated with acute functional Fe deficiency and impairments in cognition ${ }^{(22)}$. Using a substantively similar battery of cognitive performance measures as employed in the present study, Kretsch et al. ${ }^{(23)}$ found that, after 15 weeks of laboratory-controlled energy restriction, there were significant relationships between a number of blood Fe factors and task performance. Specifically, after 15 weeks of weight loss, performance on a task with a high working memory load was correlated with both $\mathrm{Hb}$ and transferrin saturation. This indicates that alterations in blood $\mathrm{Fe}$ metabolism during controlled weight loss may, at least, partially explain the observed impairments in cognitive function.

The aims of the present study were twofold. First, the study investigated whether the impairments in working memory capacity observed in unsupported $v$. supported naturalistic dieting are a replicable phenomenon. Second, the study investigated whether any observed impairments could be attributed to alterations in Fe metabolism resulting from attempted weight loss.

\section{Methods}

\section{Participants}

Participants comprised healthy, pre-menopausal, overweight women (BMI between 25 and $29 \mathrm{~kg} / \mathrm{m}^{2}$ ), aged $20-45$ years with normal or corrected to normal visual acuity and English as their first language, recruited via newspaper advertisement asking for individuals to take part in a study examining the relationship between weight status and psychological function from the Birmingham UK area. Potential participants ( $n$ 252) completed an initial telephone health screening and, on the basis of this, a postal health screening questionnaire; seventy-five participants attended their baseline session. The screening procedure was designed to exclude participants with physical or psychiatric health problems, smokers, heavy drinkers, those taking dietary supplements, oral contraceptive users, and those currently pregnant or lactating. Written consent was obtained at a pre-baseline screening session after the experimental procedures were explained to all participants. During this pre-baseline session, height and body weight were measured and self-report measures of affective state (the Hospital Anxiety and Depression Scale (HADS) ${ }^{(24)}$ ) were completed to ensure no group baseline differences in affective state $(P>0.05)$. In addition, all participants completed the Dutch Eating Behaviour Questionnaire $(\mathrm{DEBQ})^{(25)}$ in order to assess habitual eating behaviour. After the screening procedure, participants were randomised to one of three groups; these being supported dieters, unsupported dieters and a non-dieting control group. The final sample sizes for each group reflect dropout rates, which did not significantly differ between the groups $(P>0.05)$. Only the data for participants who completed the whole 8 weeks of the study are included.

\section{Design and procedure}

The present study comprised a randomised, 8-week-long controlled trial in which the effects of supported $v$. unsupported dieting on cognitive function Fe status were investigated. All participants attended three test sessions; these being a baseline session, a second session 1 week later and a third session 8 weeks post-baseline. In one group, participants attended a commercially available organised weight-loss group for this period. This commercial weight-loss plan comprised an energy-restricted, nutritionally balanced diet in conjunction with weekly weighing and group support sessions. No foods were provided to the participants. In the second group, participants were asked to pursue any diet plan they chose for 8 weeks, provided that the diet chosen did not entail weight loss as part of an organised group. The diets chosen ranged from nutritionally balanced, energy-restricted diets, low-fat diets and low-carbohydrate diets. The final group acted as non-dieting controls. Participants were randomised to one of the three groups on a rolling basis upon initial recruitment, with the intention of a final group of twenty-five. The final group sizes are the result of participant dropout after attendance at the baseline test session and failure to obtain blood samples on all sessions. Final group sizes reflect the number of participants who completed all three test sessions. Ethical approval for the study was given by the Aston University Human Research Ethics Committee.

Upon arrival at the laboratory for each session, participants were weighed in underwear and without shoes and their percentage of body fat determined using the foot-to-foot bio-impedance/body composition analyser (In-Body $220^{\mathrm{TM}}$; Derwent Healthcare). This was completed behind a screen and participants were not informed of their results until the final debriefing. Height was initially measured using a wall-mounted stadiometer (being used in conjunction with weight to provide data for BMI calculations). Each participant then donated a venous blood sample and then completed a computerised battery of neuro-psychological function tasks. These comprised a measure of vigilance performance (a visual analogue of the Bakan Vigilance Task $^{(6,7,15)}$ ), a measure of immediate verbal recall ${ }^{(6,7,15)}$, a measure of Simple Reaction Time ${ }^{(6,7,15)}$, a measure of motor speed $^{(6,7,15)}$ (Two-Finger Tapping performance), a Mental 
Rotation $\operatorname{Task}^{(7,12,15,22)}$ (week 1 test session only) and a manual, non-computerised version of the Tower of London $\operatorname{Task}^{(7,12,15,21)}$. The Tower of London Task was only administered during the second test session due to its widely known susceptibility to practice effects ${ }^{(26)}$ and the previously demonstrated finding that the effects of dieting on cognition are most marked in the first 2 weeks of attempted weight loss ${ }^{(7)}$. While not comprising a formalised neuro-psychological performance battery validated among clinical populations, these were tasks which have been previously demonstrated to show sensitivity to nutritional manipulations and dieting status, in particular. Given the hypotheses outlined concerning the effects of dieting status on specific components of the working memory system and their previously demonstrated ability to illustrate these effects ${ }^{(7,20)}$, it was judged that they were appropriate measures in order to ensure comparability with past findings.

\section{Cognitive assessment battery}

The tasks were presented to subjects via a personal computer running MEL version 2 (Psychology Software Tools, Inc.) with a $13^{\prime \prime}$ colour monitor. The tasks were presented in the same order during each session and are described below.

Two-Finger Tapping Task (motor speed). Subjects were required to alternately tap the ' 1 ' and ' 2 ' keys of the numeric keypad as quickly as possible, using the first and second fingers of the preferred hand. The dependent variable was the time taken to make 300 key presses.

Verbal Free Recall Task (phonological loop). Subjects were presented with two lists of twenty words each, one list being presented at $1 \mathrm{~s} /$ word and the other at $2 \mathrm{~s} /$ word. Immediately after the presentation of each list, subjects were given $4 \mathrm{~min}$ to recall as many words as possible from that list.

Bakan Vigilance Task (sustained attention and general working memory). This was a $6 \mathrm{~min}$ long, visual analogue of the procedure developed by Bakan ${ }^{(27)}$ in which subjects were presented with a continuous stream of single digits ${ }^{(1-9)}$ which appeared one at a time, in the centre of the visual display unit. The subjects' task was to press a labelled response key, as quickly as possible, when they believed that they had detected a sequence of either three odd numbers or three even numbers occurring consecutively. The stimulus exposure duration was $600 \mathrm{~ms}$, with no inter-stimulus interval and fortyeight potential correct targets. The dependent variable was the number of correct hits made over the course of the task.

Mental Rotation Task (visuospatial sketchpad). This task assessed the capacity of the visuospatial sketchpad component of the working memory system. Subjects were presented with a number of pairs of random geometric forms, each comprising the eight- and twelve-point shaped used by Cooper $^{(28)}$. Each pair of forms was presented simultaneously, positioned in the centre of two circles. The participants' task was to mentally rotate the form on the right-hand side of the screen until they believed it to be in the same orientation as the form on the left-hand side of the screen. They then had to decide whether they believed the form on the right-hand side of the screen to be a duplicate, or a mirrored image of the form on the left. They were instructed to respond by pressing the 1 key on the numeric keypad if they believed the two images to be duplicates and the 2 key if they believed them to be mirror images of each other. The right-hand forms either required a $0,60,120,180,240$ or $300^{\circ}$ clockwise mental rotation. In total, there were ninety-six trials, these comprising eight trials each of the two forms, in each of the possible rotation positions.

Simple Reaction Time (reaction speed). In this task, subjects were presented (via a computer visual display unit), with 100 reaction time trials (divided into five blocks of twenty trials each for the purposes of analysis) with instructions to hit the space bar, as quickly as possible, as soon as they detected a single star appearing in the centre of the monitor. There was a variable stimulus onset of either 1,3,7 or $16 \mathrm{~s}$, in order to prevent subjects anticipating the appearance of the stimulus on screen.

The Tower of London Task (central executive). This task is considered to be one of the primary means of assessing the capacity of the Central Executive component of working memory ${ }^{(29,30)}$. In the present study, participants were required to complete a manual version of this task. The task comprised a three-peg board with three moveable wooden discs, one green, one white and one black. The participants' task was to move the discs from a standard start position to a target position in a set minimum number of moves. There were two target positions that could be achieved in a minimum of two moves, two that could be achieved in three moves, four that could be achieved in four moves and four that could be achieved in a minimum of five moves. Administration of the test followed the standardised procedure outlined by Krikorian et $a l .{ }^{(30)}$. The battery was designed to assess the capacity and function of the three components of working memory and psychomotor speed.

Also, participants recorded a $3 \mathrm{~d}$ estimated food record, including one weekend day and two weekdays, during the week before baseline and again before each subsequent test session. Food records were coded and analysed for energy intake $(\mathrm{kJ})$ and using the Weighed Intake Software Program version 2.0 (Tinuviel Software). In addition, participants completed $100 \mathrm{~mm}$ visual analogue scales of somatic sensations immediately before cognitive testing on each session, these measuring 'hunger', 'thirst', 'desire to eat' and 'fullness, ${ }^{(6-8)}$. These were anchored with 'not at all' at the zero point and 'extremely' at the other end of the scale, participants being instructed to indicate how they felt 'at the moment'.

\section{Blood collections}

Fasting venous blood samples $(20 \mathrm{ml})$ were drawn from the antecubital vein using Vacuette ${ }^{\mathrm{TM}}$ (Greiner Bio-One). Blood samples for $\mathrm{Zn}$ analysis were drawn into Sarstedt syringes (Sarstedt, Inc.) containing ammonium heparin-coated beads (15 $\mu \mathrm{l}$ heparin/ml blood), placed on ice for no longer than $60 \mathrm{~min}$, and centrifuged in a Heraeus Labofuge 300 centrifuge (Kendro Laboratory Products $\mathrm{GmbH}$ ) for $10 \mathrm{~min}$ at $2400 \mathrm{rpm}$ $(1145 \mathbf{g})$. Plasma was transferred using polypropylene transfer pipettes into new polypropylene tubes. Samples were stored at $-70^{\circ} \mathrm{C}$ until analysed. 


\section{Haematology and iron analyses}

Haematological analyses were performed at the City Hospital Pathology Laboratory in Birmingham, West Midlands using a Beckman-Coulter Access 2 Analyzer (chemiluminescence assays) (Beckman Coulter Inc.) and included Hb, haematocrit, erythrocyte count, mean cell volume, mean corpuscular $\mathrm{Hb}$ and mean corpuscular $\mathrm{Hb}$ concentration. Fe status parameters included serum ferritin, serum Fe, percentage of transferrin saturation and total Fe binding capacity. Serum ferritin was determined using a chemiluminescent system (Dimension Vista 500; Siemens Healthcare Diagnostics). Serum Fe, serum transferrin and unsaturated Fe binding capacity were analysed colorimetrically using the Hitachi 902 Clinical Chemistry Analyzer (Roche Diagnostics).

\section{Vitamin $B_{12}$ analysis}

Vitamin $\mathrm{B}_{12}$ levels were analysed since it is an important cofactor in the synthesis of $\mathrm{Hb}$. Levels were determined using Advia 120's (Bayer Healthcare).

The present study was conducted according to the guidelines laid down in the Declaration of Helsinki and all procedures were approved by the Aston University Human Research Ethics Committee. Written consent was obtained during the pre-trial screening session.

\section{Statistical analyses}

All statistical analyses were performed using SPSS version 16.0 (SPSS, Inc.). Unless otherwise stated, the values in the text and tables are presented as means and standard deviations and the significance criterion used is $P<0 \cdot 05$. Post hoc analysis was completed using Fisher's least significant difference test, with Bonferroni corrections for multiple comparisons.

\section{Results}

\section{Baseline data}

The baseline screening data are shown in Table 1. One-way ANOVA, with participant group as the factor, revealed no significant group-related differences in HADS scores or DEBQ subscale scores at the outset of the study $(P>0.05)$.

Table 1. Baseline Dutch Eating Behaviour Questionnaire (DEBQ) and Hospital Anxiety and Depression Scale (HADS) scores for all three groups (Mean values and standard deviations)

\begin{tabular}{|c|c|c|c|c|c|c|}
\hline & \multicolumn{2}{|c|}{$\begin{array}{c}\text { Supported } \\
\text { dieters ( } n \text { 14) }\end{array}$} & \multicolumn{2}{|c|}{$\begin{array}{l}\text { Unsupported } \\
\text { dieters ( } n 17)\end{array}$} & \multicolumn{2}{|c|}{$\begin{array}{c}\text { Controls } \\
(n 14)\end{array}$} \\
\hline & Mean & SD & Mean & SD & Mean & SD \\
\hline \multicolumn{7}{|l|}{ DEBQ } \\
\hline Restraint & $2 \cdot 41$ & $0 \cdot 19$ & $2 \cdot 87$ & 0.09 & 2.78 & 0.21 \\
\hline External eating & 3.07 & 0.14 & 3.26 & 0.14 & $3 \cdot 15$ & 0.16 \\
\hline Emotional eating & 2.59 & 0.19 & 2.99 & $0 \cdot 13$ & 3.02 & 0.20 \\
\hline \multicolumn{7}{|l|}{ HADS } \\
\hline Anxiety & $1 \cdot 30$ & 0.01 & 2.04 & $0 \cdot 11$ & 1.76 & 0.14 \\
\hline Depression & 2.45 & 0.10 & 1.84 & 0.21 & 1.99 & 0.21 \\
\hline
\end{tabular}

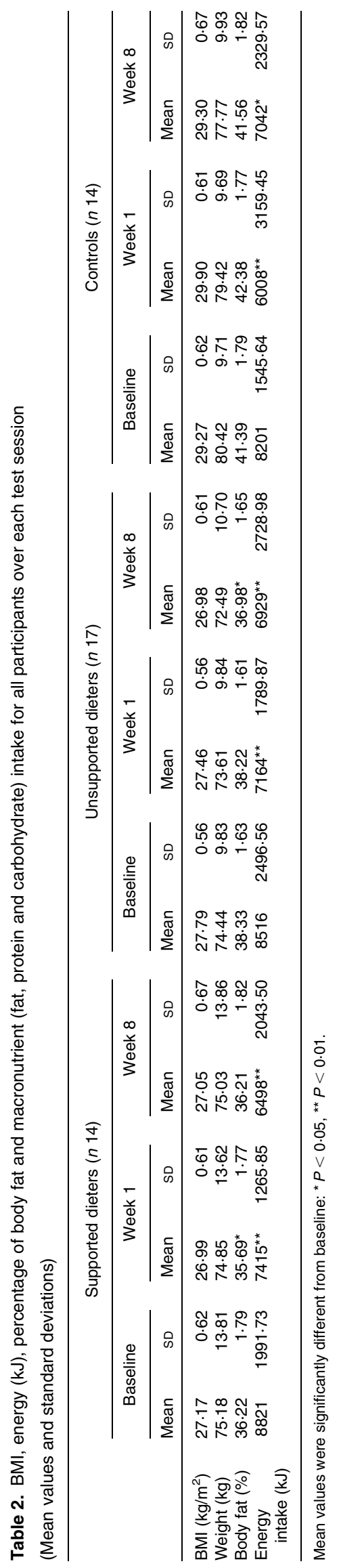




\section{Anthropometric data}

The BMI, percentage of body fat and energy intake data for each session are shown in Table 2. All data were analysed via two-way ANOVA (with participant group and test session as factors).

$B M I$. There were no significant main effects or interactions involving BMI $(P>0.05)$.

Percentage of body fat. There was a significant interaction between participant group and test session $\left(F_{(4,82)}=3 \cdot 415\right.$, $P=0 \cdot 012$ ). Post hoc analysis revealed that the supported dieters experienced a decrease in body fat between the first and second sessions, but had regained this by the time of the final session $(P<0.05)$. The unsupported dieters experienced a reduction in body fat between the baseline and final sessions $(P<0.05)$. There were no significant changes in percentage of body fat for the control group over the course of the study $(P>0.05)$.

Energy intake. There was a main effect of test session, indicating that all three participant groups reported a reduction in energy intake $\left(F_{(2,41)}=6.893, P=0.003\right)$. There were no other significant main effects or interactions $(P>0.05)$.

\section{Iron and vitamin $B_{12}$ status}

The results of the blood sample analysis are shown in Table 3. All blood results were within normal parameters.

$H b$. There was no significant group $\times$ session interaction $\left(F_{(2,82)}=0.395, P>0.05\right)$, although there was a significant main effect of session $\left(F_{(2,41)}=3.571, P=0.032\right)$, with levels being higher in the second session than in either the baseline or final session. There were no other significant main effects or interactions $(P>0.05)$.

Mean corpuscular $\mathrm{Hb}$. There was a significant interaction between test session and group $\left(F_{(2,82)}=2 \cdot 629, P=0.040\right)$. Post hoc analysis revealed that the unsupported dieters exhibited a significant decrease in mean corpuscular $\mathrm{Hb}$ over the course of the study, whereas the other two groups did not $(P<0.05)$. There were no significant main effects $(P>0.05)$.

Erythrocyte count. There was no significant group $\times$ session interaction $\left(F_{(2,82)}=1.827, P>0.05\right)$, although there was a significant main effect of session $\left(F_{(2,41)}=4.605\right.$, $P=0 \cdot 016$ ), with levels being higher in the second session than in either the baseline or final session. There were no other significant main effects or interactions $(P>0.05)$.

There were no other significant main effects or interactions for any of the other blood Fe, haematological or vitamin variables $(P>0.05)$.

\section{Cognitive function}

The data for the Bakan Vigilance, Verbal Free Recall, TwoFinger Tapping, Mental Rotation and SRT tasks are shown in Table 4.

Bakan Vigilance Task. There was a significant interaction between test session and participant group $\left(F_{(4,82)}=5 \cdot 122\right.$, $P=0 \cdot 001)$. Post hoc analyses indicated that, for the unsupported

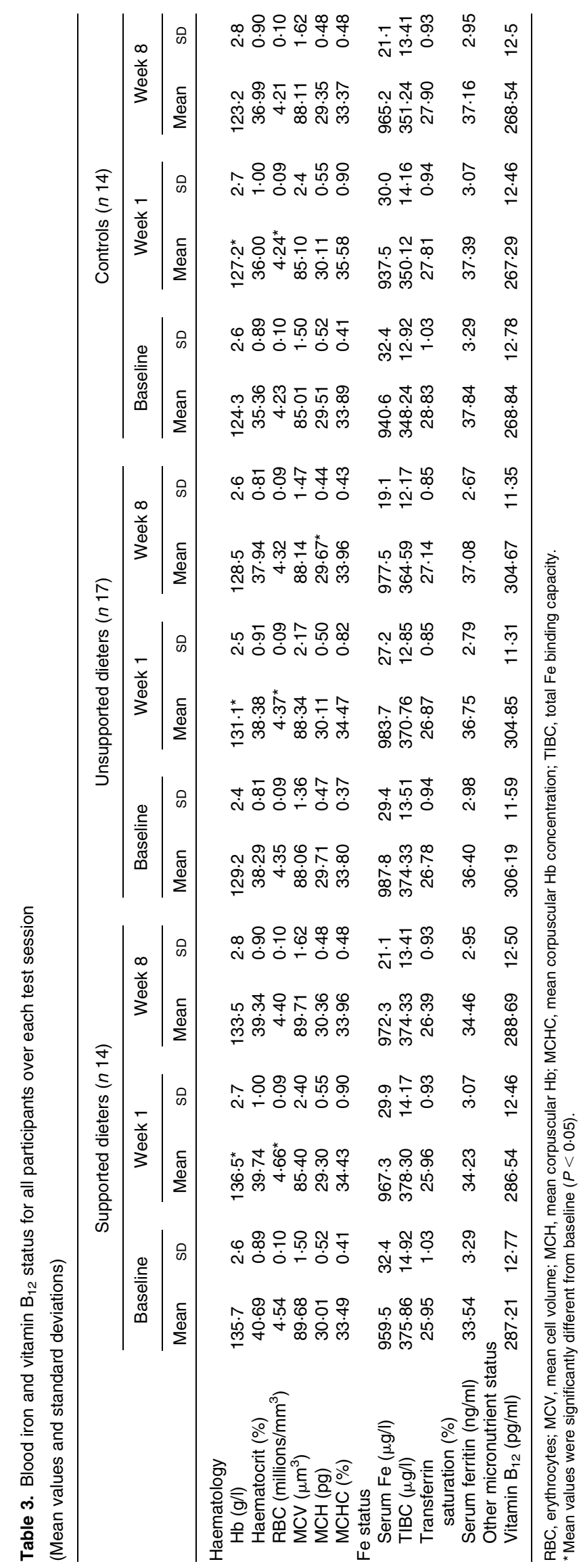


dieting group, significantly fewer correct hits were made after 1 week of attempted dieting, than either before or after $(P<0.01)$. There was also a significant main effect of time on task $\left(F_{(5,38)}=28.86, P<0.001\right)$, indicating a general decline in performance over the course of each session, consistent with the classically observed vigilance decrement ${ }^{(28)}$. There was also a significant main effect of session $\left(F_{(2,41)}=7.245, \quad P=0.002\right)$, indicating a general practice effect, with performance overall being better on the final session than at baseline.

Immediate Verbal Recall Task. There was a significant interaction between test session and participant group $\left(F_{(4,82)}=6.368, P<0.001\right)$. Post hoc analysis revealed that, for the unsupported dieting group only, performance was poorer during the second test session than during either the baseline or final test sessions $(P<0 \cdot 01)$. There was also a significant main effect of presentation speed $\left(F_{(1,42)}=22 \cdot 808\right.$, $P<0.001)$, with recall being significantly better in the $2 \mathrm{~s} /$ word presentation speed than the $1 \mathrm{~s} /$ word speed. There were no other significant main effects or interactions $(P>0.05)$.

Two-Finger Tapping Task. There were no significant main effects or interactions for tapping performance $(P>0.05)$.

Mental Rotation Task. There was a significant main effect of test session $\left(F_{(2,41)}=7.013, P=0.002\right)$, with times taken by all participant groups to make the same/mirror image decision improving with each test session, consistent with a practice effect. There was also a significant main effect of angle of rotation $\left(F_{(5,38)}=9.513, \quad P<0.001\right)$, with decision times increasing with angle of mental rotation required to make the same/mirror image decision. There were no other significant main effects or interactions $(P>0.05)$.

Simple Reaction Time Task. There was a significant threeway interaction between test session, participant group and trial block $\left(F_{(16,72)}=2 \cdot 041, \quad P=0 \cdot 022\right)$. Post hoc analysis revealed that, while reaction times generally increased over the course of each test session (consistent with a fatigue effect), this effect was significantly larger for the unsupported dieters in the second test session $(P<0.05)$. There were also significant main effects of test session and trial block $(P<0.01)$.

Tower of London Task. The planning time data for the Tower of London Task are shown in Fig. 1. There was a significant interaction between participant group and problem difficulty $\left(F_{(6,80)}=2 \cdot 517, \quad P=0.028\right)$. Post hoc analysis revealed that the unsupported dieters had significantly longer planning times for the four- and five-move problems than either the supported dieters or non-dieting control participants $(P<0.05)$. There were also significant main effects of participant group and problem difficulty $(P<0 \cdot 01)$.

\section{Somatic Sensations Questionnaire}

The results of the Somatic Sensations Questionnaire are shown in Table 5.

Hunger. There was a significant interaction between participant group and test session $\left(F_{(4,82)}=4 \cdot 678, P=0 \cdot 002\right)$. Post hoc analyses revealed that the unsupported dieters 


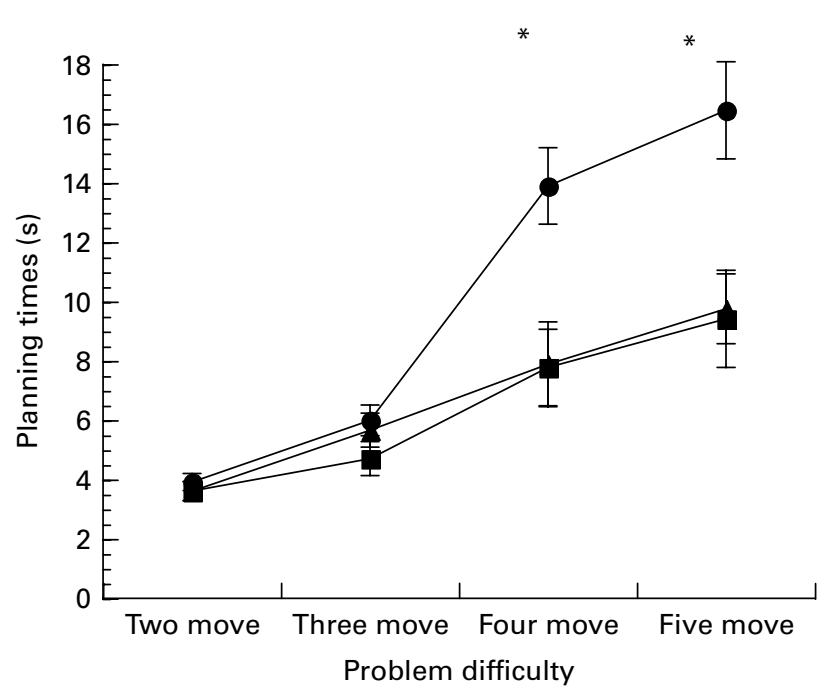

Fig. 1. Mean planning times on the Tower of London Task for all three participant groups. Values are means, with their standard errors represented by vertical bars. ${ }^{*}$ Mean values were significantly different from those of the other two participant groups $(P<0.05)$. $\rightarrow$, Supervised dieters; $\longrightarrow-$, unsupervised dieters; $\longrightarrow$, non-dieting controls.

displayed a significantly greater degree of self-reported hunger in the second test session than either at baseline or the final test session $(P<0 \cdot 01)$. There were also significant main effects of participant group $(P<0.05)$ and test session $(P<0.001)$.

Desire to eat. There was a significant interaction between participant group and test session $\left(F_{(4,82)}=7 \cdot 415, P<0 \cdot 001\right)$. Post hoc analysis revealed a significantly higher desire to eat among the unsupported dieters after 1 week of attempted weight loss $(P<0 \cdot 01)$ than during the other sessions. Desire to eat did not significantly change across the course of the studies for either of the other two test groups $(P>0.05)$. There were also significant main effects of participant group and test session $(P<0.001)$.

Fullness. There was a significant main effect of participant group $\left(F_{(2,42)}=9.933, P=0.027\right)$. Post hoc analyses showed that the unsupervised dieting group displayed lower degrees of self-reported fullness during each test session $(P>0.05)$. There was also a significant main effect of test session $\left(F_{(2,41)}=6.063, P=0.005\right)$. Post hoc analyses revealed that, overall, fullness ratings were lower on the second test session than at baseline or the final session $(P<0.05)$. There was no significant interaction between the two variables analysed $(P>0.05)$.

Thirst. There were no significant main effects or interactions involving self-reported thirst $(P>0.05)$.

\section{Relationships between task performance and other variables}

Pearson's product moment correlations (with appropriate Bonferroni corrections for multiple comparisons) were carried out between the cognitive function variables which were shown to be sensitive to the experimental manipulation and the self-report/blood analysis variables, separately for each participant group. No significant correlations were found

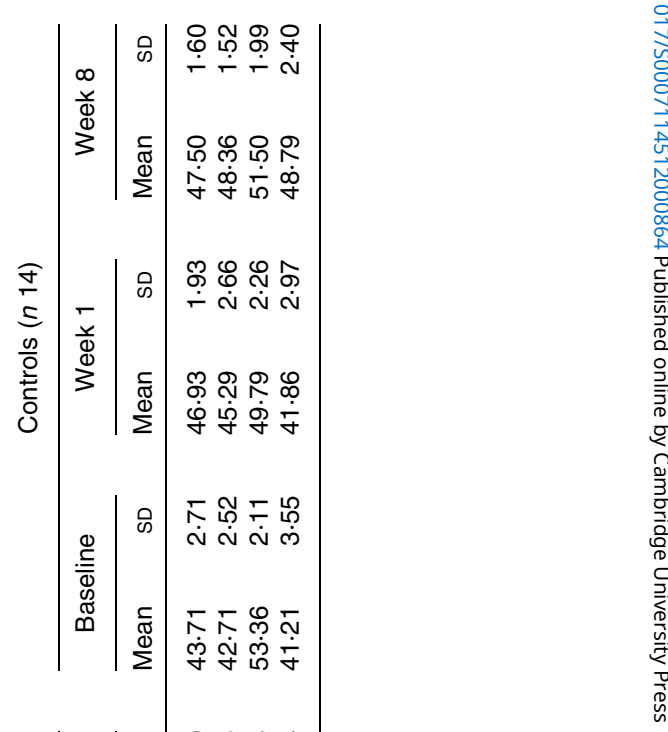

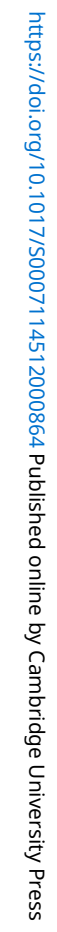


between any task performance variables and haematological/ vitamin status or anthropomorphic/somatic sensation data $(P>0 \cdot 05)$.

\section{Discussion}

The main finding of the present study was the replication of the impairment in cognitive function among unsupported dieters, relative to supported dieters or non-dieting controls, within the first week of attempted weight loss ${ }^{(7,15)}$. Primarily, the impairments appear to be in tasks which assess the capacity of the central executive and phonological loop components of working memory ${ }^{(12)}$, although simple reaction speed also appeared to be impaired in unsupported dieters during the early stages of weight loss ${ }^{(6)}$. The second main finding of the present study was that, although there were some group-specific changes in blood chemistry over the course of the study, none of these was specifically related to changes in cognitive function. In addition, changes in energy intake, BMI and percentage of body fat were unrelated to changes in cognitive function, as were changes in somatic sensations relating to eating (e.g. self-reported 'hunger' or 'desire to eat'). This latter finding does not support previous findings ${ }^{(8,9,12,13)}$, which have indicated that the primary cause of dieting-related impairment in cognitive function is the result of preoccupying cognitions relating to food preferentially consuming limited working memory capacity. The present data do not, however, preclude the possibility that the observed effects of dieting on cognitive function are the result of preoccupying cognitions relating to body shape or self-esteem $^{(11-13)}$. Any future attempt at replication of the present study should include a measure of body shape-related self-esteem such as the Body Attitudes Questionnaire ${ }^{(30)}$. The finding of no relationship between task performance and BMI, percentage of body fat and energy intake is consistent with other findings ${ }^{(7)}$ and is suggestive of the dietingrelated cognitive impairments being due to psychological, rather than physiological, factors. This conclusion is further supported by the finding that impaired task performance was only observed among the unsupported dieters and only during the first week of attempted weight loss, with performance returning to levels comparable to those of the other two participant groups by the final test session.

The main significant finding relating to Fe status was that participants in the unsupervised dieting group experienced a decrease in mean corpuscular $\mathrm{Hb}$ over the course of the study which was not found in the other two groups. This was, however, unrelated to cognitive function, weight or energy intake and, during each test session, was still within the normal healthy range. This is in contrast to studies which have demonstrated that very-low-energy, experimentally controlled weight loss induces negative changes in a number of $\mathrm{Fe} /$ haematological variables and that these are related to relative impairments in cognitive function ${ }^{(22,23)}$. The present study demonstrates that the cognitive impairments associated with non-laboratory-based, real-world, unsupported dieting are not the result of compromised Fe metabolism. Reasons for the difference in cognitive function between the two groups after 1 week of attempted weight loss are unclear. Potential reasons may include the use of structured diet plans, group support and regular weighing in the presence of others in the supported diet group. These should be the subject of further research. The results also showed that all three participant groups reported progressively smaller energy intakes over the course of the study, but with no corresponding change in BMI. A possible reason for this apparently contradictory finding lies in reliance on the accuracy of self-reported energy intake, which has shown itself to not be completely accurate. For instance, a number of studies have found serious under-reporting biases with such dietary records ${ }^{(31-33)}$. Future attempts at verifying the present study should attempt to verify the accuracy of self-reported energy intake using the doubly labelled water technique.

In conclusion, the present data highlight the need to make a clear distinction between the effects of laboratory-induced food restriction and dieting behaviour outside of the laboratory $^{(5)}$. While the two forms of energy restriction appear to exert a negative effect on brain function, it is by no means clear that the causal mechanisms of these impairments are the same in both situations.

\section{Acknowledgements}

The authors would gratefully like to acknowledge the assistance of Dr Stuart Williams, of the Department of Pathology, City Hospital Birmingham with the analysis of the blood samples obtained during the course of this study. This research received no specific grant from any funding agency in the public, commercial or not-for-profit sectors. Both authors contributed equally to the screening data collection and analysis. M. W. G. prepared the manuscript. The authors confirm that there is no conflict of interest.

\section{References}

1. National Center for Health Statistics (2006) Chartbook on Trends in the Health of Americans Health, United States, 2006. Hyattsville, MD: Public Health Service.

2. Bray G (1996) Health hazards of obesity. Endocrin Metab Clin 25, 907-919.

3. Department of Health (2008) Healthy Weight, Healthy Lives: A Cross Government Strategy for England (2008). London: UK Government.

4. Warren C \& Cooper PJ (1988) Psychological effects of dieting. Br J Clin Psych 71, 247-261.

5. Green MW (2010) Cognitive performance deficits of dieters. In The International Handbook of Behaviour, Diet and Nutrition [VR Preedy, RR Watson and CR Martin, editors]. New York: Springer.

6. Green MW, Rogers PJ, Elliman NA, et al. (1994) Impairment of cognitive processing associated with dieting and high levels of dietary restraint. Physiol Behav 55, 447-452.

7. Green MW \& Rogers PJ (1995) Impaired cognitive function during spontaneous dieting. Psychol Med 25, 1003-1010.

8. Green MW, Elliman N \& Rogers PJ (1997) Impaired cognitive processing in dieters: failure of attentional focus or resource capacity limitation? Br J Health Psych 2, 259-267. 
9. Kemps E, Tiggemann M \& Marshall K (2005) Relationship between dieting to lose weight and the functioning of the central executive. Appetite 45, 287-294.

10. Kemps E \& Tiggemann M (2005) Working memory performance and preoccupying thoughts in female dieters: evidence for a selective central executive impairment. Br J Clin Psych 44, 357-366.

11. Shaw J \& Tiggemann M (2004) Dieting and working memory: preoccupying cognitions and the role of the articulatory control process. BrJ Health Psych 9, 179-185.

12. Green MW \& Rogers PJ (1998) Impairments in working memory associated with spontaneous dieting behaviour. Psychol Med 28, 1063-1070.

13. Vreugdenburg L, Bryan J \& Kemps E (2003) The effect of self-initiated weight-loss dieting on working memory: the role of preoccupying cognitions. Appetite 41, 291-300.

14. Baddeley AD (1986) Working Memory. Oxford: Oxford University Press.

15. Green MW, Elliman NA \& Kretsch MJ (2005) Weight loss strategies, stress and cognitive function: supervised vs unsupervised dieting. Psychoneuroendocrinology 30, 908-918.

16. Green MW, Elliman NA \& Rogers PJ (1995) A lack of effect of short-term food deprivation on cognitive function. J Psychiat Res 29, 245-253.

17. Kretsch MJ, Green MW, Fong AKH, et al. (1997) Cognitive effects of a long-term weight reducing diet. Int $J$ Obes Relat Metab Disord 21, 14-21.

18. Green MW, Elliman NA \& Rogers PJ (1997) The effects of food deprivation and incentive motivation on blood glucose levels and cognitive function. Psychopharmacology $\mathbf{1 3 4}$, 88-94.

19. Anderson IM, Parry-Billings M, Newsholme EA, et al. (1990) Dieting reduces plasma tryptophan and alters 5-HT function in women. Psychol Med 2, 785-791.

20. Green MW, Jones AD, Smith I, et al. (2003) Impairments in working memory associated with naturalistic dieting in women: no relationship with urinary 5-HIAA levels? Appetite 40, 145-153.
21. Kretsch MJ, Blanton CA \& Green MW (2006) Cognitive impairment in low-iron status premenopausal women. FASEB J 20, A1003.

22. Beard J, Borel M \& Peterson J (1997) Changes in iron status during weight loss with very low energy diets. Am J Clin Nutr 66, 104-110.

23. Kretsch MJ, Fong AKH, Green MW, et al. (1998) Cognitive function, iron status and haemoglobin concentration in obese dieting women. Eur J Clin Nutr 52, 512-518.

24. Snaith RP \& Zigmnd SS (1994) The Hospital Anxiety and Depression Scale Manual. Windsor: NFER-Nelson.

25. Van Strien T, Frijters JER, Bergers GPA, et al. (1986) The Dutch Eating Behavior Questionnaire (DEBQ) for assessment of restrained, emotional and external eating behavior. Int J Eat Disorder 5, 295-315.

26. Beauchamp MH, Dagher A, Aston JAD, et al. (2003) Dynamic functional changes associated with cognitive skill learning of an adapted version of the Tower of London task. Neuroimage 20, 1649-1660.

27. Bakan P (1959) Extroversion-introversion and improvement in an auditory vigilance task. Br J Psychol 50, 323-332.

28. Cooper LA (1975) Mental rotation of random twodimensional shapes. Cog Psych 7, 20-43.

29. Shallice T (1982) Specific impairments of planning. Philos Trans $R$ Soc Lond B 298, 199-209.

30. Krikorian R, Bartok J \& Gay N (1994) Tower of London procedure: a standard method and developmental data. J Clin Exp Neuropsych 16, 840-850.

31. Ben-Tovim DI \& Walker MK (1991) The development of the Ben-Tovim Walker Body Attitudes Questionnaire (BAQ), a new measure of women's attitudes towards their own bodies. Psychol Med 21, 775-784

32. Livingstone MBE, Prentice AM, Strain JJ, et al. (1990) Accuracy of weighed dietary records in studies of diet and health. Br Med J 300, 708-712.

33. Krall EA \& Dwyer JT (1987) Validity of a food frequency questionnaire and a food diary in a short-term recall situation. J Am Diet Assoc 87, 1374-1377. 